\title{
Service Cost Estimation in Cloud Environment Using a Third Party Web Server: A Comparative Analysis With and Without Using Cloud Computing
}

\author{
Rashmi Ranjan Dhal ${ }^{1}$ \& B V A N S S Prabhakar Rao ${ }^{2}$ \\ ${ }^{\prime}$ (M.Tech-CSE Cloud Computing, VIT University, India) \\ ${ }_{2}^{2}$ Asst. Professor(SG), School of Computing Science and Engineering, VIT University, India)
}

\begin{abstract}
This paper maps the idea of software maintenance cost estimation process onto cloud computing service cost. We have many models for effort estimation in maintenance. Here we implement any cost estimation model to calculate the service cost in cloud computing. As in cost model there are 14 major cost drivers, in cloud environment there are also cost drivers which hold major expenditure. Considering cloud environment cost drives, an effort estimating equation is developed which can be implemented for finding the service cost. Using that service cost a third party server gives back pre-deployment maintenance estimation to the cloud user on request. There is also an analysis of data sets from different organizations which shows the comparative cost involved in cloud services and in traditional services.
\end{abstract}

Keywords: Cloud, Total Bandwidth Cost, Total Instance Cost, Total Storage Cost, Total Cost Ownership

\section{INTRODUCTION}

Software development and maintenance are two major aspects of software development lifecycle whether it may be Waterfall model or it may be Spiral model. Maintenance of software by the developer for the customer takes $70-80 \%$ budget of total investment for the software from the requirement analysis phase because sometimes changes of software are so sensitive that a small change costs double of initial investment. For effort calculation in maintenance process many analysts gave many equations like Boehm's ACT model, COCOMO model, FP model etc. When we move onto effort estimation part we have to point out all the factors which demand charges [1]. In traditional service model, a customer has to order for the software service and availability of that software is checked and then it is notified to the customer. Then after all the payment and license issue the product is delivered to the customer by media like CD, floppy disk, etc. After getting the software the customer installs it on its local system. Here the pricing units are the media cost, transportation cost, local hardware cost, installation cost, update and upgrade cost. Moving to the web based model, the service provider of a particular software posts all the products on the web. The customer only makes the payment and gets the license of that product and installs in local hardware. Here there is no media and transportation cost, only the cost of the product, local hardware cost and update and upgrade cost. Finally coming to the cloud service which is the recent trend where every software service, resource have been already placed on ASP site. The customer uses directly without any costly hardware. Everything is managed and upgraded by the professional of Host [7].

\section{IMPORTANCE OF MAINTENANCE}

Maintenance is needed because from time to time the requirement of customer changes, the market requirement changes, the platform of implementation changes; so software has to work in all upcoming environments to meet the above requirements. Software estimation is sometimes confused with size and effort. What is the size in software development context is a complete set of business functionalities that the end users get when the product is deployed and used and also the number of persons involved in the cloud server.

2.1 .Adaptive Maintenance: Cloud Web Service (e.g. Amazon EC2) should provide a specific platform which is going to satisfy all the changing demands of the customer. It's likely to adapt the environment.

2.2 .Preventive Maintenance: The server inside the cloud prevents the upcoming error or problem like overwhelming of access over bandwidth. System backup should be maintained on regular basis in the form of different servers and database to prevent data loss. Security alert should be given frequently by the cloud service provider so that the user is always aware of virus and hacker.

2.3 .Enhancement Maintenance: Enhanced version of service should not conflict with existing features. Enhancement of software is done when it is needed. When the number of users increases, the server capacity as well as the database capacity should be enhanced [7]. 


\section{Cloud Computing As MaintenanCe Agent}

Cloud is a package having lots of data centers which consist of a large number of computers to provide services to the user. If the user wants an application to run on his system, he has to buy it ,install it , and run it; and if he wants some more features than the existing one, then he has to go for maintenance. But cloud computing technique eases all these activities starting from buying of software package to using software and maintaining that software. Considering the IaaS aspect of cloud computing like network server, OS, processor which are inside the cloud, the user only gets the access on payment to the service where maintenance is done by the cloud manager himself. So the need of the customer and what platform he is going to use have to be specified and up to that requirement the changed service is served to the customer. Cloud also acts as the storage or backup of program and application. As per the user's requirement, it resizes itself. A large data backup should be done with minimal interface. That back up of Virtual Machine is attached with virtual disk. This back up workload is assigned to proxies [8].

\section{Methodology}

We may think about an exchange server. The cloud manager has to install OS security patches. Software rollups installed, database maintenance, hardware maintenance, and other critical support system related to main flow- firewalls, anti spam and antivirus -need to be looked after as well. This can be done when we create server instance in cloud. All the traditional security patching and software updating are required to maintain a healthy server capable of servicing the client. Here we describe two methodologies which can find the cost of service in cloud computing. First approach is inference analysis method. In this method, some information about the previous similar project's estimation is required and usually the estimation process in these methods is done according to the analysis of the previous datasets. Here we can calculate all the individual or atomic cost, then we sum it up to find the total cost or we can derive the cost of service from overall general properties, then we split it up to different components.

Second approach is mathematical approach or algorithmic approach. We have to bring out all the major cost drivers and make those as the variable of a cost estimating function $(\mathrm{F})$ :

$\mathrm{E}=\mathrm{F}(\mathrm{y} 1, \mathrm{y} 2, \ldots \mathrm{y}(\mathrm{n}-1), \mathrm{yn})$

Where $\mathrm{E}$ is the effort for estimation and $\mathrm{y} 1, \mathrm{y} 2$,etc are the cost drivers like COCOMOII. Generally we divide the cost factor into 3 types:(i) Storage,(ii)Platform, and (iii) Bandwidth.

\section{Cost Estimation Process}

We have to detect the factors which demand charge, and then we can move onto estimating the cost for overall process. First step is to look for deployment cost. It means initially how much resource is allocated to the system for service. If extra resource is required in-between the service period, the charge for the same is included in overall cost.

Pricing Unit: Instance usage is charged at per hour basis means duration and input/output traffic is usually charged at memory basis i.e. per GB usage and backup or storage is charged per memory requirement basis means per tera-byte. So different IaaS service providers opt different pricing models for charging. Hence due to complexity a general pricing model is decided and total duration of using software is calculated for network traffic.

5.1. Pricing for Storage: When a user uses the service, he doesn't know the software specification. So a document containing the inter communication among them is required because at running time of that software it may require additional memory like $1 \mathrm{~GB}$ or $1 \mathrm{~TB}$. To meet this requirement additional memory is needed over the local storage memory. If a user is using a particular service which demands extra memory at some particular time which is different from normal usage then that pricing band falls in different category. Because previously used standard instance storage, bandwidth allowance and charging rate are different. So there should be a single pricing band for the whole total usage for the additional storage.

5.2. Boundary of Network: Different pricing models are deployed for different resource allocation in different places. Bandwidth calculation depends on what traffic comes into services means what traffic is generated and distributed within service. So to calculate the bandwidth usage network boundary concept is generated. We can calculate incoming and outgoing traffic with this mechanism.

5.3.Data transfer count: How much data is transferred between resources can be monitored by a resource connection point. So we can calculate the network bandwidth usage. 


\section{MODEL FOR COST ESTIMATION}

For designing a cost model first we have to analyze the user requirement i.e. what type of service the user wants e.g. use of application, storage, network, etc. Then from that the cost demanding individual units are analyzed. We have to specify the charges associated with those units. These facts are described in user specification document, cloud description and software specification document. Gathering all those factors an equation for finding out the total cost can be developed. After that we go for implementation of that equation as an algorithm to design a cost estimating model

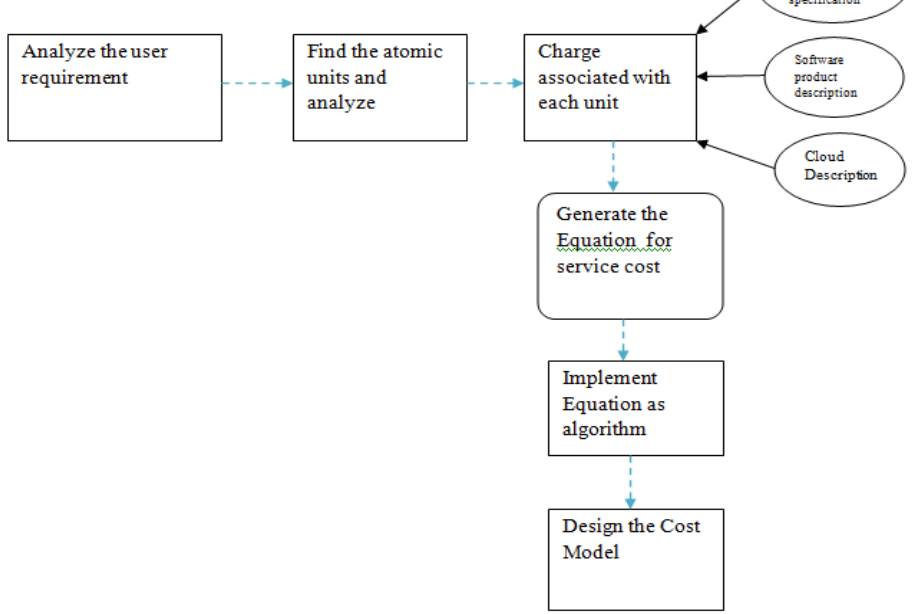

Fig-1: Service Cost Estimation Model

VII. Cost Model Details

Here we describe the various cost types and their description so that we can proceed to design a cost model for service delivery.

\section{Factors of Cost estimations}

\begin{tabular}{|l|l|}
\hline Total Instance Cost & $\begin{array}{l}\text { Virtual Machine Type, Machine Cost, } \\
\text { Number of amount, Service type }\end{array}$ \\
\hline Total Bandwidth Cost & $\begin{array}{l}\text { No. of VM, type of connector, No. of port, } \\
\text { Type of Port, Types of Port, No. of Port. }\end{array}$ \\
\cline { 2 - 3 } & Data in, Data out \\
\cline { 2 - 2 } & $\begin{array}{l}\text { Machine, Region, Cloud service, IaaS } \\
\text { Provider }\end{array}$ \\
\hline & Cloud Service Type \\
\hline & $\begin{array}{l}\text { Computing power, Storage Capacity, } \\
\text { Inbound Data Transfer, Outbound Data } \\
\text { Transfer }\end{array}$ \\
\hline & $\begin{array}{l}\text { Basic Service Charge, Internal Data } \\
\text { Transfer }\end{array}$ \\
\hline Total Storage Cost & $\begin{array}{l}\text { Duration, Data Input } \\
\text { Table-1: Cost Details } \\
\text { VIII. Cost Model }\end{array}$ \\
\hline
\end{tabular}

The cost model assumes the total cost to be the sum of all the 3 cost factors. That can be represented as $\boldsymbol{T C} \boldsymbol{E}=\sum \boldsymbol{e}^{\boldsymbol{C T}}$ Where $\mathrm{CT}$ is the cost types. $\mathbf{C T}=\sum \boldsymbol{C}_{\boldsymbol{F}}^{\boldsymbol{C T}}$. F:cost factors.

Cost model considers the duration as the period of time for which the cloud service is being used. As it's on hourly basis, the Total usage time $T_{T U}=T_{D} \times 24$ as minimum duration is on day basis.

1.Total Instance Cost(TIC):

$$
\mathrm{TIC}=N_{\text {Machine }}^{\text {Size }} \times T_{T U} \times P_{\text {instance }}
$$

$\boldsymbol{P}_{\text {instance }}$ is retrieved from cloud description document, Number of machine size $\left(\boldsymbol{N}_{\text {Machine }}^{\text {Size }}\right)$ is extracted from software product description, Duration $\left(\boldsymbol{T}_{\boldsymbol{T U}}\right)$ is extracted from user requirement description document. 
2.Total Bandwidth Cost(TBC):The bandwidth cost depends on the amount of data transferred from and to the port applying the input\& output boundary price that transfers data from single component. Boundary price is extracted from cloud description document. The calculation also includes a cost of total data input amount into the system which is extracted from user requirement description document.

$$
\begin{gathered}
D T_{\text {to }}^{\text {Port }} \rightarrow \text { data transferred to the port } \\
D T_{\text {from }}^{\text {Port }} \rightarrow \text { data transferredfrom the port } \\
D_{\text {in }}^{\text {price }} \rightarrow \text { data in price } \\
D_{\text {out }}^{\text {price }} \rightarrow \text { data out price }
\end{gathered}
$$

$$
\mathrm{TBC}=D T_{\text {to }}^{\text {Port }} \times D_{\text {in }}^{\text {price }}+D T_{\text {from }}^{\text {Port }} \times D_{\text {out }}^{\text {price }}
$$

3.Total Storage Cost(TSC): It depends on the total storage amount and the price associated with this. Storage amount ( $\boldsymbol{T}_{\text {Storage ammnt }}$ ) is extracted from software product description document and storage price $\left(\boldsymbol{P}_{\text {storage }}\right)$ is extracted from cloud description document. So the total storage cost is defined below

$$
\mathrm{TSC}=\boldsymbol{T}_{\text {Storage ammnt }} \times \boldsymbol{P}_{\text {storage }}
$$

Total Cost Estimation: It is the sum of total instance cost, total bandwidth cost and total storage cost.

\section{TCE$=\mathbf{T I C}+\mathrm{TBC}+\mathrm{TSC}$}

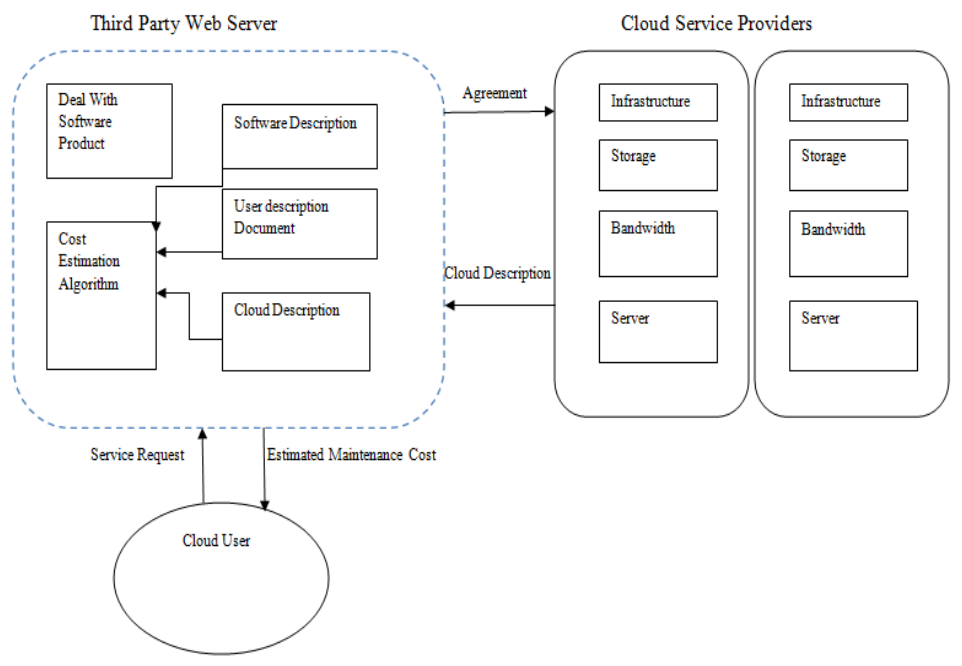

Fig-2: Third Party Web Server Model

After getting the cost of all the units, we design a model which estimates the maintenance effort using the above equation. It uses a third party who manages this maintenance related activity. First, pre service deployment request is sent by cloud user to the third party web server. All the requests are maintained in user description document in XML format. Then the third party makes a business deal with cloud service providers and gets the cloud description and software product description according to the user's request. Then it gathers all the numerical data from the documents and applies the cost estimation algorithm to find estimated cost. Then the user gets the pre- deployment maintenance cost.

\section{Case Study}

A case study shows us that VMware Virtualization software reduces the maintenance cost. The Total Cost Ownership methodology considers 3 sectors like Healthcare, Insurance and Transportation. It analyzes major two aspects that contribute to TCO of server like hardware and software, and IT operations. It shows a graphical representation of impact of virtualization on those three sectors. 


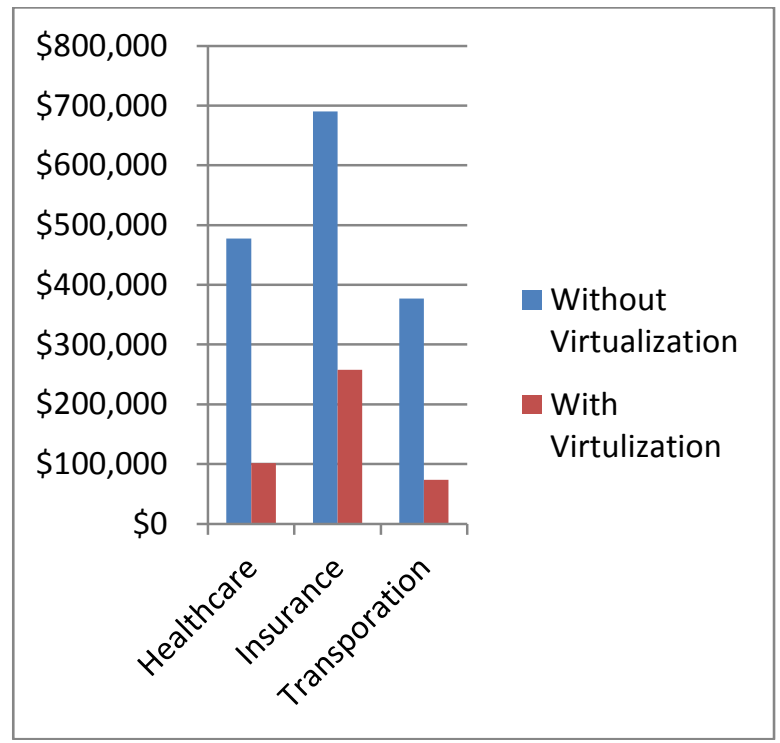

\section{Conclusion}

All the organizations across the globe understand the maintenance process to properly maintain the server, system, database and all other equipment in cost-effective way. So they can put lesser number of staff for maintenance.

We can also analyze the features of TABWARE cloud computing and asset management. It provides a maintenance program without upfront cost and there is also no planning requirement for maintenance. All the database backup and accidental cost are handled by Asset Point. By this Tabware many organizations move towards productivity with maximizing performance and minimizing maintenance cost.

\section{XI. $\quad$ Future Work}

This model only allows calculating the service cost. We can put this model as a basis and develop separate algorithms which would calculate consecutively for storage usage, network usage, etc. For resource, a Resource Allocation Based Model and for network traffic we can develop Data Usage Model. Similarly if our service is on time basis then the model will keep track of time slice and for the cost factor another algorithm will be designed. After all these, analyzing the record of all process, a virtual analytical model can be designed which can predict the total cost for maintenance without running the service.

\section{Journal Papers:}

\section{REFERENCES}

[1] Juan Carlos Granja-Alvarez and Manuel Jose Barranco-Garcia, Software Maintenance:Research and Practice,1997, Vol. 9, 161175

[2] Pragya Siddhi, Varun Kumar Rajpoot, A Cost Estimation of Maintenance Phase for Component Based Software, IOSR Journal of Computer Engineering,ISSN : 2278-0661 Volume 1, Issue 3 (May-June 2012), PP 01-08

[3] Sugandha Chakraverti, Prof Sheo Kumar, Dr. S.C. Agarwal, Ashish Kumar Chakraver, Modified Cocomo Model For Maintenance cost Estimation of Real Time System Software „IJCSN, Volume 1, Issue 1, February 2012

[4] Yi-Kuei Lin 1, and Ping-Chen Chang, Estimation of Maintenance Reliability for a Cloud Computing Network, International Journal of Operations Research Vol,2010. 7, No. 1, pp 53-60

[5] Yunsik Ahn, Jungseok Suh, Seungryeol Kim and Hyunsoo Kim, Journal of Software Maintenance and Evolution: Research and Practice, 2002;15:71-85.

[6] Arshdeep Bahga ,Vijay K. Madisetti, Analyzing Massive Machine Maintenance Data in a Computing Cloud, IEEE Transactions on Parallel and Distribured Systems, vol. 23, no. 10, Ocober 2012

[7] Mengmeng Liu, Zachary G. Ives, and Boon Thau Loo. Enabling incremental query re-optimization. Under submission to Proceedings of the ACM SIGMOD International Conference on Management of Data, Scottsdale, Arizona, USA, 2012.

[8] Mengmeng Liu, Nicholas E. Taylor, Wenchao Zhou, Zachary G. Ives, and Boon Thau Loo. Maintaining recursive views of regions and connectivity in networks. IEEE Trans.Knowl. Data Eng., 22(8):1126\{1141, 2010.

[9] Aggarwal, K.K., Gupta, J.S. and Misra, K.B. (1975). A simple method for reliability evaluation of a communication system.IEEE Transactions on Communications, 23, 563-566.

[10] Alexopoulos, C. (1995). A note on state-space decomposition methods for analyzing stochastic flow networks. IEEETransactions on Reliability, 44, 354-357.

[11] Aven, T. (1985). Reliability evaluation of multistate systems with multistate components. IEEE Transactions on Reliability, 34, 473-479

[12] Bodin, L.D., Golden, B.L., Assad, A. A. and Ball, M.O. (1982). Routing and scheduling of vehicles and crews: the state of the art. Computers and Operations Research, 10, 63-211. 
[13] R. Jiang and D. N. P. Murthy, "Modeling failure-data by mixture of Weibull distributions: a graphical approach," IEEE Transactions on Reliability, vol. 44, no. 3, pp. 477-488, 1995

[14] M. Xie and C. D. Lai, "Reliability analysis using an additive Weibull model with bath-tub shaped failure rate function," Reliability Engineering and System Safety, vol. 52, pp. 87-93, 1996.

\section{Books:}

[15] Gopalaswamy Ramesh \& Ramesh Bhattiprolu, Software Maintenance (Tata McGraw - Hill Education,2005,India ).

[16] Rajib mall, Fundamentals of Software Engineering (PHI Learning Private Limited ,2009, India)

[17] Kai Hwang, Geoffrey Fox, Jack J. Dongarra, Morgan Kaufmann, Distributed and Cloud Computing: From Parallel Processing to the Internet of Things, 1st Edition, 2011.

[18] Gautham Shroff, Enterprise Cloud Computing: Technology, Architecture, Applications, Cambridge press, 2010.

[19] Kris Jamsa, Cloud Computing, Jones \& Barlett Learning, 2013

[20] Rajkumar Buyya, James Broberg, Andrzej Goscinski,Cloud Computing Principles and Paradigms, John Wiley \& Sons, 2011 